\title{
Resistant Hypertension in Hemodialysis Patients
}

\author{
Authors \\ Ahmad B. Elden', Walaa H. Mohamad', Muhammad Hosam Magraby ${ }^{3}$ \\ ${ }^{1,3}$ Critical Care Unit, Assiut University, ${ }^{2}$ Nephrology Unit, Assiut University \\ Corresponding author
}

Ahmad B. Elden

Assiut University, P.O. Box 71111 Assiut, Egypt

Telephone: +20-1009820300, Fax: +20-2333327

Email:ahmadbahie@yahoo.com

\begin{abstract}
Objectives: This study aimed to assess prevalence of resistant hypertension (RHT) in hemodialysis (HD) patients.

Patients and Methods: We conducted a cross sectional study on 135 HD patients. RHT was defined as failure to reach target blood pressure (BP) control (systolic BP $<140 \mathrm{mmHg}$ and diastolic BP $<90 \mathrm{mmHg}$ ) with maximal dose of 3 antihypertensive therapies (AHT) including diuretics in patients with good daily urine output (> $500 \mathrm{cc} /$ day) or at least $2 \mathrm{~L} /$ session ultrafiltration volume in oliguric (<500-100 cc/day) or anuric patients (<50 cc /day). Patients with post HD hypertension underwent 24 ambulatory monitor for their BP, which hourly measured their BP.

Results: In our study; 34 (25.2\%) of patients were non hypertensives, 43 (31.8\%) were controlled hypertensives, $58(43.0 \%)$ were uncontrolled hypertensives. Uncontrolled hypertensives with inadequate AHT were 53 (39.2\%), where RHT patients were 5 (3.7\%) of all study population.

Conclusions: We concluded that RHT had3.7\% prevalence in HD patients.

Keywords: resistant hypertension, haemodialysis.
\end{abstract}

\section{Introduction}

Hypertension is a major contributor toexcessive cardiovascular morbidity and mortality in in hemodialysis (HD) patients because it is a cause as well as a consequence ofchronic kidney disease (CKD) and end-stage renal disease (ESRD ${ }^{[1]}$. Multiplemechanisms are likely involved in blood pressure dysregulation in HD patients and some of themhave resistant hypertension.RHT, defined as
BP that remains above goal despite treatment with 3 different classes of AHTagents. One of the 3 agents should be a diuretic and all agents should be prescribed at optimal dose amounts orcontrolled hypertension with at least 4 drugs $^{[2]}$.

Aggressivecontrol of hypertension in dialysis ismandatory.Antihypertensive therapy (AHT) in HD patients was associated with a reduced risk of cardiovascular events, all-cause mortality, and 
cardiovascular mortality ${ }^{[3]}$. HD provides better volume control inESRDpatients byachieving patient's dry weight. However, clearly defined guidelines are not available for hypertensive patients in the HD population and nearly $50-60 \%$ of HD patients continue to suffer fromHTN ${ }^{[4]}$ despite multiplicity of AHT.RHT is estimated to affect $15 \%$ to $30 \%$ of patients with essential hypertension ${ }^{[5]}$. The prevalence of RHT in dialysis patients is still unidentified. Our aim of this study was tostudy the prevalence of RHT in HD patients.

\section{Patients and methods}

We conductedacross sectional study, carried out on 135 patients who undergo regular $\mathrm{HD}$ in theDialysis Unit of Assiut University Hospital in 2015. Written consents were obtained from most of the participants; illiterate participants gave their consent by finger prints. The study was approved by the ethical committee of Facultyof Medicine in Assiut University. Blood pressurewas measured by well calibrated mercurial sphygmomanometers in 3 different haemodialysis sessions, half an hour before, one hour during and half an hour after cession.Recruited patients on AHT instructed to take their medications at night and to collect their urine output in sterile secured bottles. We used JNC 7 criteria for diagnosis of hypertension with systolic $\mathrm{BP} \geq 140 \mathrm{mmHg}$ and/or diastolic $\mathrm{BP} \geq 90$ $\mathrm{mmHg}$. Patients with post dialysis hypertension underwent $24 \mathrm{~h}$ ambulatory monitor for their BP usingDel Mar Reynolds/England S.N; 00008970, which hourly measuredtheir BP. Maximal, minimal systolic and diastolic BPs were calculated. Night non dipper patients were identified. RHT was defined as failure to reach target BP control (systolic BP $<140 \mathrm{mmHg}$ and/or diastolic $\mathrm{BP}<90 \mathrm{mmHg}$ ) with maximal dose of 3 medications including diuretics in patients with good daily urine output (> $500 \mathrm{cc} /$ day) or at least $2 \mathrm{~L} /$ session ultrafiltration volume in oliguric $(<500-100 \mathrm{cc} /$ day $)$ or anuric patients $(<50 \mathrm{cc}$ /day).

\section{Classification of patients}

We classified our recruit according to their hypertension state into 3 groups; non hypertensives (NonHTN) with pre, intra and post dialysis $\mathrm{BP}<140 \mathrm{mmHg}$ and diastolic $\mathrm{BP}<90$ $\mathrm{mmHg}$ ) without AHT, controlled hypertensives $(C H T N)$ with pre, intra and post dialysis BP $<140$ $\mathrm{mmHg}$ and diastolic BP $<90 \mathrm{mmHg}$ ) with AHT, and uncontrolled hypertensives groups (UCHTN)with pre, intra and post dialysis BP $\geq 140 \mathrm{mmHg}$ and or diastolic $\mathrm{BP} \geq 90 \mathrm{mmHg}$ ) with AHT. UCHTN was sub classified into UCHTN with inadequate AHT (less than 3 AHT) and RHTas previously defined.

\section{Statistical analysis}

The statistical analysis wasperformed using SPSS (version 16.0, SPSS Inc., Chicago, IL, USA). The Kolmogorov-Smirnov test will be used to test normally. The continuous variables were presented as the means \pm SDand categorical variables were presented as percentages. Student $T$ testwas used to compare between means of 2 continuous variables, ANOVA test was used to compare between means of $>2$ continuous variables, and chi square testwas used to compare percentages and ratios. A p-value $<0.05$ was considered statistically significant

\section{Results}

\section{Basal characteristics}

The studywas carried out on 135 regular HDpatients.34 (25.2\%) of patients were NonHTN, $43(31.8 \%)$ were $C \mathrm{HTN}, 58 \quad(43.0 \%)$ were UCHTN (Figure 1). There were insignificant differences between groups as regard age, gender, BMI, apparent aetiology of ESRD and duration of dialysis (Table 1).

There were insignificant differences between $C H T N$ and UCHTN groups in duration of hypertension. $C H T N$ had significantly higher urine output than $U C H T N$. 
Table (1):Demographic, Selected Clinical and Laboratory Characteristics of Patients

\begin{tabular}{|c|c|c|c|c|c|}
\hline CHARACTERISTICS & & $\begin{array}{l}\text { Non HTN } \\
(n=34)\end{array}$ & $\begin{array}{l}\text { CHTN } \\
(n=43)\end{array}$ & $\begin{array}{l}\text { UCHTN } \\
(n=58)\end{array}$ & (P Value) \\
\hline AGE(YEARS) & & $42.35 \pm 15.46$ & $43.60 \pm 12.89$ & $42.41 \pm 13.55$ & 0.396 \\
\hline MALEN (\%) & & $20(58.8 \%)$ & $24(55.8 \%)$ & $24(41.4 \%)$ & 0.187 \\
\hline \multirow[t]{4}{*}{ BMI } & Underweight N (\%) & $6(17.6 \%)$ & $2(4.7 \%)$ & $3(5.2 \%)$ & 0.064 \\
\hline & Normal weight $\mathrm{N}(\%)$ & $18(52.9 \%)$ & $22(51.2 \%)$ & $30(51.7 \%)$ & 0.988 \\
\hline & Overweight $\mathrm{N}(\%)$ & $7(20.6 \%)$ & $9(20.9 \%)$ & $11(19.0 \%)$ & 0.966 \\
\hline & Obese $N(\%)$ & $3(8.8 \%)$ & $10(23.3 \%)$ & $14(24.1 \%)$ & 0.169 \\
\hline \multirow[t]{4}{*}{ Etiology } & Diabetes N (\%) & $7(20.6 \%)$ & $13(30.2 \%)$ & 24 (41.4\%) & 0.112 \\
\hline & HypertensionN (\%) & $0(0.0 \%)$ & $6(14.0 \%)$ & $4(6.9 \%)$ & 0.066 \\
\hline & CIN N (\%) & $21(61.8 \%)$ & $18(41.9 \%)$ & $22(37.9 \%)$ & 0.074 \\
\hline & Others N (\%) & $6(17.6 \%)$ & $6(14.0 \%)$ & $8(13.8 \%)$ & 0.865 \\
\hline Duration of dialysis(YEARS) & & $5.81 \pm 4.40$ & $5.30 \pm 4.09$ & $4.10 \pm 3.17$ & 0.087 \\
\hline $\mathrm{HB}(\mathrm{g} / \mathrm{dl})$ & & $10.5 \pm 2.39$ & $11.0 \pm 3.15$ & $10.1 \pm 2.22$ & 0.453 \\
\hline Serum $\mathrm{Ca}(\mathrm{mg} / \mathrm{dl})$ & & $8.1 \pm 2.08$ & $8.6 \pm 2.13$ & $8.3 \pm 1.52$ & 0.314 \\
\hline Serum phosphate(mg/dl) & & $4.0 \pm 0.72$ & $3.9 \pm 0.91$ & $4.01 \pm 0.69$ & 0.608 \\
\hline $\operatorname{PD} \mathbf{B U N}(\mathrm{mg} / \mathrm{dl})$ & & $55.30 \pm 5.30$ & $53.9 \pm 7.2$ & $55.0 \pm 0.02$ & 0.355 \\
\hline
\end{tabular}

NonHTN;non hypertensives, CHTN; controlled hypertensives, UCHTN; uncontrolled hypertensives,BMI: body mass index, ESRD: end stage renal disease, CIN: chronic interstitial nephritis, CIN included;chronic pyelonephritis, obstructive uropathy, analgesic nephropathy, reflux nephropathy, others included;ADPCKD, chronic GN, and SLE, PN BUN; post dialysis Blood Urea Nitrogen

There were insignificant differences between groups in rate and amount of ultrafiltration. $C H T N$ had significantly lower BP measurements (pre, intra and post dialysis) and lower numbers of AHT than UCHTN group.

Uncontrolled hypertensives with inadequate AHT

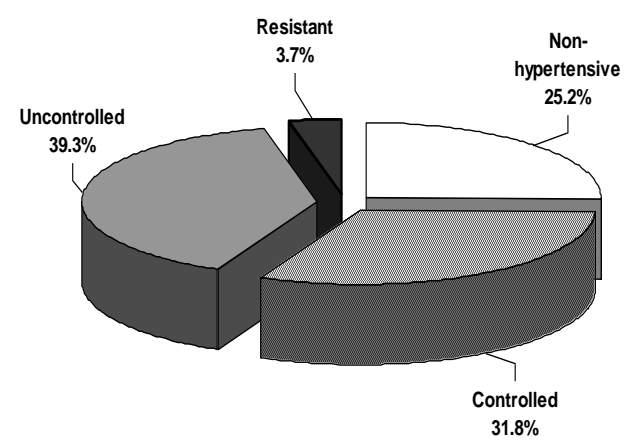

Figure 1: distribution of hypertensive state were $27(46.6 \%)$ with only one AHT and 26 (44.8\%) with two AHT, with a total of 53 (91.4 $\%$ ) of UCHTN and $39.2 \%$ of all study population. RHT patients were $5(3.7 \%)$ of all study population (Figure 1).

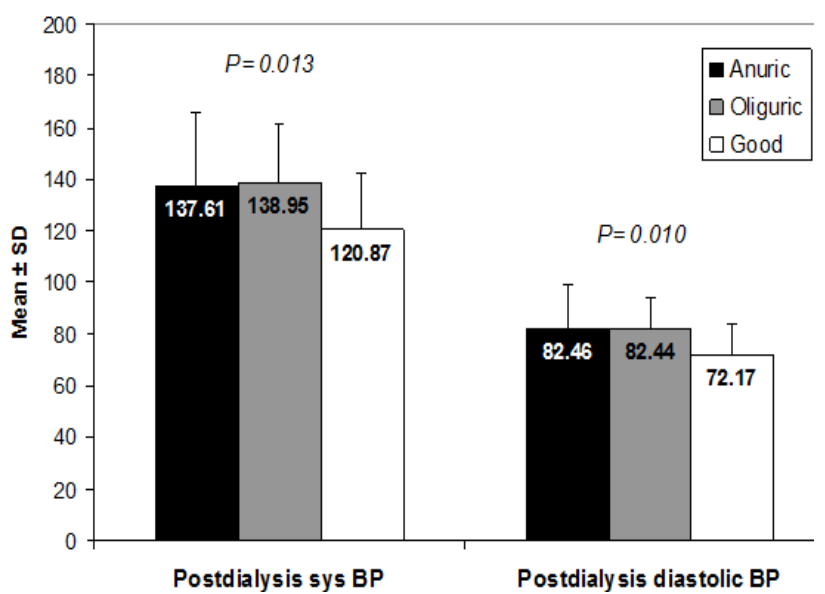

Figure 2:comparing post dialysis BP in urine output groups 


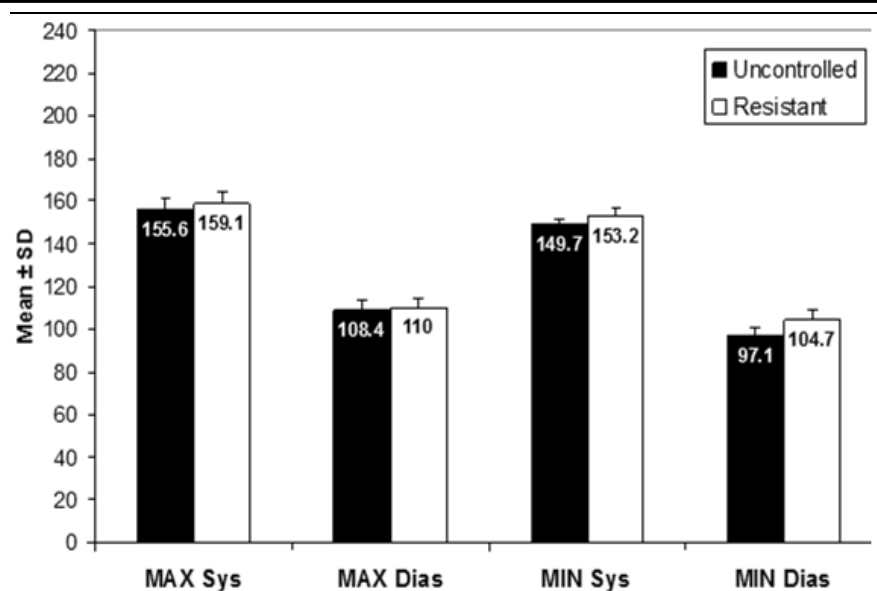

Figure 3: comparisons between $\mathrm{BP}$ in resistant hypertensives and other uncontrolled hypertensives
BP: blood pressure, MAX: maximum, Sys: systolic, MIN: minimal, Dias: diastolic
There were insignificant differences between uncontrolled hypertensives with inadequate AHTandRHT regarding; mean of maximal systolic pressure, mean of maximal diastolic pressure, mean of minimal systolic pressure, mean of minimal diastolic pressure (Figure3) and nondipper state (Not shown).

Table (2):Hypertension duration, urine output,ultrafiltration characters and drug therapies

\begin{tabular}{|c|c|c|c|c|}
\hline CHARACTERISTICS & & $\begin{array}{l}\text { CHTN } \\
(n=34)\end{array}$ & $\begin{array}{l}\text { UCHTN } \\
(n=58)\end{array}$ & P-value \\
\hline DURATION OF HTN (YEARS) & & $7.58 \pm 5.62$ & $7.38 \pm 7.34$ & 0.881 \\
\hline \multirow[t]{3}{*}{ URINE OUTPUT } & Anuric & $19(44.2 \%)$ & 31 (53.4\%) & \multirow{3}{*}{0.016} \\
\hline & Oliguric & $12(27.9 \%)$ & $23(39.7 \%)$ & \\
\hline & Good & $12(27.9 \%)$ & $4(6.9 \%)$ & \\
\hline ULTRAFILTRATION & & $10(23.3 \%)$ & $21(36.2 \%)$ & 0.163 \\
\hline ULTRAFILTRATION VOLUME & & $3.00 \pm 0.67$ & $3.38 \pm 0.92$ & 0.253 \\
\hline \multirow[t]{2}{*}{ PRE DIALYSIS } & Systolic BP (mmhg) & $125.58 \pm 19.25$ & $148.71 \pm 25.44$ & 0.000 \\
\hline & Diastolic BP (mmhg) & $77.33 \pm 18.72$ & $85.86 \pm 13.93$ & 0.010 \\
\hline \multirow[t]{2}{*}{ INTRA DIALYSIS } & Systolic BP (mmhg) & $121.40 \pm 13.55$ & $151.64 \pm 20.42$ & 0.000 \\
\hline & Diastolic BP (mmhg) & $74.65 \pm 17.91$ & $88.79 \pm 11.41$ & 0.000 \\
\hline \multirow[t]{2}{*}{ POST DIALYSIS } & Systolic BP(mmhg) & $116.28 \pm 9.77$ & $153.79 \pm 19.52$ & 0.000 \\
\hline & Diastolic BP (mmhg) & $70.00 \pm 8.73$ & $90.26 \pm 10.61$ & 0.000 \\
\hline \multirow[t]{3}{*}{ NUMBER OF DRUGS } & One Drug & $32(74.4 \%)$ & $27(46.6 \%)$ & \multirow{3}{*}{0.015} \\
\hline & Two Drugs & $8(18.6 \%)$ & $26(44.8 \%)$ & \\
\hline & Three Drugs & $3(7.0 \%)$ & $5(8.6 \%)$ & \\
\hline
\end{tabular}

ANURIC: < $100 \mathrm{cc} /$ day, OLIGURIC: 500 -100 cc/day and GOOD: > 500cc/day.

\section{Urine output and post dialysis BP}

By pooling both $C H T N$ and UCHTN groups, there was a significant trend of post dialysis BP to be reduced as urine output increase (Figure 2).

\section{Discussion}

Our study groups were homogenous in age, gender, apparent aetiology of ESRD, duration of dialysisand duration of hypertensionwhich reduced confounding factors. Although rate and volumeof ultrafiltration had insignificant differences betweenCHTN and UCHTN, urine output was significantly higher in $C H T N$, which may emphasize the importance of residual renal function (RRF)inBP control.RRF has been found to be important in maintaining fluid balance of 
HD patients. In the CANUSA Study, urine volume was a strong independent predictor of survival. Every $250 \mathrm{ml} / \mathrm{min}$ urine output was associated with a $36 \%$ reduction in overall mortality ${ }^{[6]}$.Loss of RRF is independently associated with suboptimal blood pressure control, likely a result of chronic volume expansion ${ }^{[7]}$.The severity of left ventricular hypertrophy $(\mathrm{LVH})$, a strong independent predictor of mortality in dialysis patients, inversely correlates with the presence of $\mathrm{RRF}^{[8]}$. In addition, loss of RRF is associated with more severe anemia, hypoalbuminemia, and higher arterial pressure ${ }^{[9]}$. Patients on HD can preserve RRF and achieve better control of their BP by using synthetic membranes and ultrapure dialysis water, routine use of ACEIs and ARBs unless contraindicated, avoid use of non-steroidal anti-inflammatory drugs, oral phosphate solutions, and prolonged aminoglycoside antibiotics, intravenous contrast $^{[10]}$.

In our study survey;we found that inadequatecontrol of hypertensionhad a more significant prevalence in HD patients than RHT, where HD with inadequate AHT accounted for 53 (39.2\%) of all study populations andRHT patients were $5(3.7 \%)$ of all hemodialysis studypopulation. Rahman et aldemonstrated that the prevalence of uncontrolled hypertension in HD patientswas $62 \%{ }^{[11]}$.

Interdialytic weight gain, as patient noncompliance with salt and fluid intake, was independent predictors of pre-dialysis systolic blood pressure ${ }^{[11]}$.Other factors contributing to inadequate control of hypertension are:underlying secondary form of hypertension, patient noncompliance on AHT due to developments of side effects andinadequate ultrafiltration ${ }^{[1]}$, inadequate prescription of medicationsdue to fear of intra-dialysis hypotension interfering with adequate ultrafiltration.

Additional strategies to reduce blood pressure should be implemented alongside the dialysis plan including; prolonged or increased frequency of HD, sympathetic denervation, bilateral nephrectomy ${ }^{[12]}$.

\section{Conclusion}

We concluded that inadequatecontrol of hypertensionhad a more significant prevalence in HD patients than RHT, where UCHTN with inadequate AHT accounted by 53 (39.2\%) of all study populations and RHT patients were 5 (3.7\%) of all hemodialysis study population and 5 (8.6\%) of UCHTN. Urine output was significantly higher in CHTN, which may emphasise the importance of RRF in BP control.

\section{Study limitations}

We recruited only Caucasian patients, which may limit extrapolation to other ethnic groups. Moreover, our results apply only to HDpatients under regular tertiary care. The small number of RHT made statistical difficulties to compare between them and $U C H T N$. We recommend the development of a well definite AHT protocols in managing hypertension in HD to aid in decreasing prevalence of uncontrolled hypertension.

\section{Acknowledgements}

The authors wish to acknowledgeDr. Abdel Mohsen Abdelhie, Mrs. Asmaa Abdellatif and Mr. Mohamoud in the dialysis unit for their contribution in gathering data and follow up of patients.

\section{Conflict of interests}

The authors declare that there was no conflict of interests as regard the publication of this paper.

\section{References}

1- Venkata C., RamS. and Andrew Z. Fenves:Management of Hypertension in Hemodialysis Patients.Current Hypertension Reports 2009, 11:292-98

2- Calhoun DA, Jones D, Textor S, et al: Resistant hypertension: diagnosis, evaluation, and treatment. A scientific statement from the American Heart Association Professional Education Committee of the Council for High Blood Pressure Research. Hypertension 
2008;51(6):1403-19.

3- Heerspink HJL, Ninomiya T, Zoungas S, de Zeeuw D, Grobbee DE and Jardine MJ, et al: Effect of lowering blood pressureon cardiovascular events and mortality in patients on dialysis:A systematic review and meta-analysis of randomised controlledtrials. Lancet 2009; 373: 1009-15.

4- Henrich WL and Lionel U. Mailloux: In: Schwab SJ, et al. eds. Hypertension in hemodialysis patients. Waltham, MA: Up to Date2013.

5- Daugherty S.L., Powers J.D. and Magid D.J:Incidence and prognosis of resistant hypertension in hypertensive patients. Circulation 2012;125:1635-42.

6- Bargman JM, Thorpe KE and Churchill DN: Relative contribution of residual renal functionand peritoneal clearance to adequacy of dialysis: a reanalysis of the CANUSAstudy. Journal of American Society of Nephrology 2001, 12 (10): 2158-62.

7- Konings CJ, Kooman JP, Schonck M, Struijk DG, Gladziwa U, Hoorntje SJ, van der Wall Bake AW, van der Sande FM and Leunissen KM: Fluid status in CAPD patients isrelated to peritoneal transport and residual renal function: Evidence from alongitudinal study. Nephrology Dialysis Transplant 2003; 18(4): 797-803

8- Wang AY, Wang M, Woo J, Lam CW, Lui SF, Li PK and Sanderson JE: Inflammation, residual renal function, and cardiac hypertrophy are interrelated and combine adversely to enhance mortality and cardiovascular death risk of peritoneal dialysis patients. Journal of American Society of Nephrology2004, 15(8): 2186-94.

9- Pecoits-Filho R, Heimburger O, Barany P, Barany P, Suliman M, Fehrman-Ekholm I, Lindholm B and Stenvinkel P: Associations between circulating inflammatorymarkers and residual renal function in CRF patients. American Journal of Kidney Disease 2003;
41(6): 1208-12.

10- Jeffrey Thomas and Isaac Teitelbaum: Preservation of Residual Renal Function in Dialysis Patients.Advances in Peritoneal Dialysis 2011; 27.

11- Rahman M, Dixit A, Donley V, Gupta S, Hanslik T, Lacson E, Ogundipe A, Weigel K and Smith MC:Factors associated with inadequate blood pressure control in hypertensive hemodialysis patients.American Journal of Kidney Disease 1999, 33(3):498506.

12- Nabela Enam, Kavita Kakkad, Akshay Amin and Carole Lever:Management of hypertension in the hemodialysispopulation: a review of the literature. Journal of Community Hospital Internal Medicine Perspectives. 2014; 4(3): 10.3402/jchimp.v4.24055. 